IRSH 50 (2005), pp. 555-557 DOI: I0.1017/S0020859005002270

(C) 2005 Internationaal Instituut voor Sociale Geschiedenis

\title{
REFEREES 2002-2004
}

We have called upon a number of referees who have often made very substantial contributions to the quality of our articles. Their efforts are genuinely appreciated. We list below the referees who have volunteered their services in 2002-2004.

\begin{tabular}{ll} 
Abrahamian, Ervand & De Vries, David \\
Alexander, Peter & Delius, Peter \\
Amark, Klas & Digby, Anne \\
Atabaki, Touraj & Dubofsky, Melvyn \\
Baer, Marc & Emsley, Clive \\
Balfour, Sebastian & Eyerman, Ron \\
Bayat, Asef & Faroqhi, Suraiya \\
Beckert, Sven & Fielding, Steven \\
Behal, Rana & Fieseler, Beate \\
Beier, A.L. & Fishman, Nina \\
Beilharz, Peter & Foner, Eric \\
Bell, Wendell & Foster, Kevin \\
Bennoune, Mahfoud & Fourcaut, Annie \\
Berg, Maxine & Fowler, David \\
Binner, Rolf & Frances, Rae \\
Blaazer, David & Frank, Christopher \\
Blom, Hans & Gile, Paul A. \\
Boomgaard, Peter & Graham, Helen \\
Boris, Eileen & Greene, Julie \\
Buchanan, Tom & Griffiths, Trevor \\
Buschak, Willy & Gumbrell McCormick, Rebecca \\
Campbell, Alan B. & Halpern, Rick \\
Carew, Tony & Hazareesingh, Sudhir \\
Casanovas, Joan & Hensel, Silke \\
Celik, Zeyneb & Heron, Craig \\
Cerutti, Simona & Herzberger, David K. \\
Chandavarkar, Rajnarayan & Hippel, Wolfgang von \\
Chase, Malcolm & Hoerder, Dirk \\
Cobble, Dorothy Sue & Hollis, Patricia \\
Cooper, Fred & Honey, Michael \\
Coquery-Vidrovitch, Cathérine & Jütte, Robert \\
Crompton, Rosemary & Kaplan, Temma \\
Cunningham, Hugh & Katainen, Elina \\
Davies, Sam & Kater, Michael H. \\
& \\
& \\
\hline &
\end{tabular}


Kaur, Amarjit

Kerr, Ian

Kessler, Mario

Kett, Joseph F.

Kirby, David

Kirk, Neville

Kjeldstadli, Knut

Kössler, Reinhardt

Kok, Jan

Kramer, Paul

Kulikoff, Allan

Lamparter, Wilfried E.

Langkau, Götz

Laybourn, Keith

Lehmbruch, Gerhard

Linder, Marc

Lockman, Zachary

Lovejoy, Paul

Lucassen, Jan

Lucassen, Leo

Lutz, Helma

Lyddon, Dave

Maas, Ineke

MacDermid, Jane

McDermott, Kevin

McIlroy, John

McKee, Chrisopher

McKibbin, Ross

Masjuan, D. Eduardo

Mastboom, Joyce M.

Melling, Joseph

Metzer, Jacob

Miles, Andrew

Misgeld, Klaus

Moch, Leslie Page

Mommsen, Hans

Montgomery, David

Moors, Annelies

Moya, Jose

Munck, Ronaldo

Niedhart, Gottfried

Olssen, Erik

Ottanelli, Fraser

Oxley, Deborah
Palmer, Bryan

Pan-Montojo, Juan

Peled, Yoav

Pérez Ledesma, Manuel

Persson, Lennart K.

Pestoff, Victor

Phimister, Ian

Polasky, Janet L.

Prellwitz, Jens

Price, Richard

Prost, Antoine

Randall, Adrian

Ratz, Ursula

Roediger, David

Rojahn, Jürgen

Ronsin, Francis

Rosenzweig, Roy

Roth, Karl Heinz

Ruotsila, Markku

Schlumbohm, Jürgen

Schmidt, Ariadne

Schultz, Helga

Sharpe, Pamela

Shpayer-Makov, Haia

Siegelbaum, Lewis $\mathrm{H}$.

Simonson, Birger

Skoutelsky, Rémi

Smail, John

Smith, Steve A.

Stokes, Lawrence D.

Studer, Brigitte

Stuurman, Siep

Swaan, Abram de

Te Brake, Wayne

Thane, Pat

Thorpe, Wayne

Tomich, Dale

Tortella Casares, Gabriel

Tosstorff, Reiner

Tunçay, Mete

Van der Walt, Lucien

Van Goethem, Geert

Walkowitz, Daniel J.

Weber, Hermann 
Weinhauer, Klaus

Yuen, Tai

Weitz, Eric D.

Zarinebaf-Shar, Fariba

Wrigley, Chris

Zelnik, Reginald

In the period 2002-2004, the following colleagues have contributed annotations to the bibliography: Kees Rodenburg, Huub Sanders, and Els Wagenaar. 